Article

\title{
Increasing Antimicrobial Resistance in Surgical Wards at Mulago National Referral Hospital, Uganda, from 2014 to 2018-Cause for Concern?
}

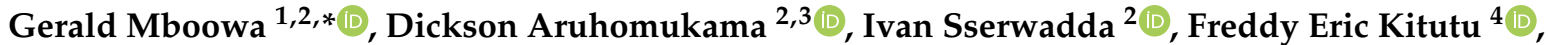 \\ Hayk Davtyan ${ }^{5}{ }^{(0)}$, Philip Owiti ${ }^{6}$, Edward Mberu Kamau ${ }^{7}{ }^{(}$, Wendemagegn Enbiale ${ }^{8,9}$, Anthony Reid ${ }^{10}$, \\ Douglas Bulafu $^{11}{ }^{1}$, Jeffrey Kisukye ${ }^{3}$, Margaret Lubwama ${ }^{3}$ and Henry Kajumbula ${ }^{3}$
}

1 The African Center of Excellence in Bioinformatics and Data-Intensive Sciences, The Infectious Diseases Institute, Makerere University, Kampala P.O. Box 22418, Uganda

2 Department of Immunology and Molecular Biology, College of Health Sciences, Makerere University, Kampala P.O. Box 7072, Uganda; dickson.aruhomukama@chs.mak.ac.ug (D.A.); ivangunz23@gmail.com (I.S.)

3 Department of Medical Microbiology, College of Health Sciences, Makerere University, Kampala P.O. Box 7072, Uganda; jkis8751@gmail.com (J.K.); maggienalum@gmail.com (M.L.); henrykajumbula427@gmail.com (H.K.)

4 Strengthening Pharmaceutical Systems (SPS) Unit, Pharmacy Department, School of Health Sciences, Makerere University, Kampala P.O. Box 7072, Uganda; kitutufred@gmail.com

check for updates

Citation: Mboowa, G.;

Aruhomukama, D.; Sserwadda, I.;

Kitutu, F.E.; Davtyan, H.; Owiti, P.;

Kamau, E.M.; Enbiale, W.; Reid, A.;

Bulafu, D.; et al. Increasing

Antimicrobial Resistance in Surgical Wards at Mulago National Referral

Hospital, Uganda, from 2014 to

2018-Cause for Concern? Trop. Med. Infect. Dis. 2021, 6, 82. https:// doi.org/10.3390/tropicalmed6020082

Academic Editors: Olga Perovic, Tom Decroo, Chakaya Muhwa Jeremiah and John Frean

Received: 22 March 2021

Accepted: 28 April 2021

Published: 19 May 2021

Publisher's Note: MDPI stays neutral with regard to jurisdictional claims in published maps and institutional affiliations.

Copyright: (c) 2021 by the authors. Licensee MDPI, Basel, Switzerland. This article is an open access article distributed under the terms and conditions of the Creative Commons Attribution (CC BY) license (https:/ / creativecommons.org/licenses/by/ $4.0 /)$.
5 Tuberculosis Research and Prevention Center NGO, Yerevan 0014, Armenia; haykdav@gmail.com

6 Academic Model Providing Access to Healthcare (AMPATH), P.O. Box 9505, Eldoret 30100, Kenya; philip.owiti@gmail.com

7 Research Capacity Strengthening, Special Programme for Research and Training in Tropical Diseases, 1201 Geneva, Switzerland; kamaued@who.int

8 Department of Dermatovenerology, College of Medicine and Health Sciences, Bahir Dar University, Bahir Dar P.O. Box 1996, Ethiopia; wendaab@gmail.com

9 Amsterdam UMC, Academic Medical Centre, Department of Dermatology, Amsterdam Institute for Infection and Immunity (AI\&I), University of Amsterdam, 1012 Amsterdam, The Netherlands

10 Médecins Sans Frontières, Operational Centre Brussels, Operational Research Unit, 1617 Luxembourg, Luxembourg; Tony.Reid@brussels.msf.org

11 Department of Environmental Health Sciences, College of Health Sciences, Makerere University, Kampala P.O. Box 7072, Uganda; bulafudouglas@gmail.com

* Correspondence: gmboowa@gmail.com; Tel.: +256-775-766-249

Abstract: Antimicrobial Resistance (AMR) and Healthcare Associated Infections (HAIs) are major global public health challenges in our time. This study provides a broader and updated overview of AMR trends in surgical wards of Mulago National Referral Hospital (MNRH) between 2014 and 2018. Laboratory data on the antimicrobial susceptibility profiles of bacterial isolates from 428 patient samples were available. The most common samples were as follows: tracheal aspirates $(36.5 \%)$, pus swabs $(28.0 \%)$, and blood $(20.6 \%)$. Klebsiella $(21.7 \%)$, Acinetobacter $(17.5 \%)$, and Staphylococcus species (12.4\%) were the most common isolates. The resistance patterns for different antimicrobials were: penicillins (40-100\%), cephalosporins (30-100\%), $\beta$-lactamase inhibitor combinations (70-100\%), carbapenems (10-100\%), polymyxin E (0-7\%), aminoglycosides (50-100\%), sulphonamides (80-100\%), fluoroquinolones (40-70\%), macrolides (40-100\%), lincosamides (10-45\%), phenicols $(40-70 \%)$, nitrofurans $(0-25 \%)$, and glycopeptide $(0-20 \%)$. This study demonstrated a sustained increase in resistance among the most commonly used antibiotics in Uganda over the five-year study period. It implies ongoing hospital-based monitoring and surveillance of AMR patterns are needed to inform antibiotic prescribing, and to contribute to national and global AMR profiles. It also suggests continued emphasis on infection prevention and control practices (IPC), including antibiotic stewardship. Ultimately, laboratory capacity for timely bacteriological culture and sensitivity testing will provide a rational choice of antibiotics for HAI.

Keywords: antimicrobial resistance (AMR); trends; Structured Operational Research and Training IniTiative (SORT IT); surgical wards; Mulago National Referral Hospital (MNRH); Uganda 


\section{Introduction}

Healthcare Associated Infections (HAIs) are a global health challenge for patient safety, and are a major driver of antimicrobial resistance (AMR) [1], particularly in lowerand middle-income countries (LMICs) [2]. Studies in Ethiopia and Nigeria have reported HAI cumulative incidences in surgical wards of between $5.7 \%$ and $45.8 \%$ [3]. Another study of 410 patients at a large hospital in northern Uganda documented an overall HAI prevalence of $28 \%$ [4]. Prevalence of HAI was higher (47\%) among surgical patients, and wound infection was three times higher among patients on emergency than elective surgery [4]. Furthermore, a study involving 83 patients with postoperative clinical surgical site infections from June to August 2015, in Mbarara regional referral hospital, southwestern Uganda, documented that Gram-negative bacteria (GNB) were predominant $(65.59 \%)$, of which Klebsiella species were $29.03 \%$ [5]. Additionally, $86 \%$ of the aerobic bacteria isolated were multidrug resistant (MDR) [5]. All the isolates, with exception of Enterococci species, were resistant to ampicillin, while GNB showed high resistance to ceftriaxone, sulfamethoxazole/trimethoprim, and gentamicin [5]. Appropriate empirical treatment of bacterial infections contracted in hospital necessitates an understanding of local AMR trends, which are only attainable through regular monitoring and surveillance.

AMR is a global health and development threat which requires urgent multi-sectoral action in order to achieve the United Nations Sustainable Development Goals (SDG), including improving global public health (SDG-3) [6]. The World Health Organization (WHO) declared AMR to be one of the top three global public health threats facing humanity, and has indicated that the misuse and overuse of antimicrobials is a principal driver in the development of drug-resistant pathogens [6]. Knowledge of local and regional AMR profiles is important for clinical decision making to improve antimicrobial use, and this can only be achieved through a regular surveillance capacity which remains largely inadequate throughout East Africa [7].

Addressing HAIs relies on timely feedback of results from AMR surveillance, and is strongly recommended by $\mathrm{WHO}$ as part of the core components of effective national infection prevention and control (IPC) programs [8]. Effective IPC is good for both HAIs and AMR prevention. HAIs contribute significantly to morbidity and mortality, lengthened hospital stay for infected patients, and, as a result, increase costs associated with healthcare [9-11]. Acquisition of HAIs depends on several factors, including patient demographics, geographical location, and post-operative hospitalisation [9-12]. They are also a consequence of the failure of antibiotics to treat infections due to AMR. In LMICs, hospital laboratories often are unable to provide culture and sensitivity testing and timely reporting of the results, so clinicians must choose antibiotics empirically. The choice for empirical antimicrobial management of HAI depends on up-to-date knowledge of local AMR patterns for the antibiotics available in that setting [9-11].

Since 2018, the Uganda AMR National Action Plan (NAP) has been implemented to prevent, slow down, and control the spread of antibiotic resistant organisms [13]. However, there are no regular country or hospital-wide monitoring and surveillance programs of AMR, as are recommended by the WHO [14]. Importantly, a number of AMR research and surveillance programs have been established and implemented; namely, Drivers of Resistance in Uganda and Malawi (DRUM), which investigates aspects of behaviour that are most important in spreading antibiotic resistance by surveying human behaviour in relation to antibiotics, water, sanitation, hygiene, and bacterial behaviour in response to these stimuli [15], as well as the UK's Fleming Fund in AMR surveillance, which aims to increase the quantity and quality of data available to enable researchers to better understand the scale and scope of AMR in Uganda [16]. In 2020, Uganda had an estimated population of 45.7 million, according to the United Nations [17]. The country's health spending per capita in 2019 was USD 44.00 [18] and Gross Domestic Product per capita was USD 956.90 [19]. The three most common causes of death in Uganda were: (i) communicable, maternal, neonatal, and nutritional diseases, (ii) non-communicable diseases, and (iii) injuries [20]. Given the prominence in Uganda of the Mulago National Referral Hospital 
(MNRH), there are no AMR trend analyses performed to date, so we studied AMR patterns in the surgical wards from 2014 to 2018 with a view to providing information about better choices of appropriate antibiotics in clinical situations.

Specifically, we aimed to describe common HAI bacterial pathogens, including Klebsiella spp., E. coli, Proteus spp., Enterobacter spp., Citrobacter spp., Serratia marcescens, Providencia spp., Acinetobacter spp., Pseudomonas spp., Staphylococcus spp., Enterococcus spp., Streptococcus spp., and Corynebacteria, to assess resistance patterns for specific antibiotics and describe trends in antibiotic resistance in the surgical wards of MNRH from 2014 to 2018.

\section{Materials and Methods}

\subsection{Study Design}

This was a descriptive cross-sectional study. We extracted a total of 428 culture and sensitivity results from five consecutive years (2014 to 2018) from the Laboratory Information Management System (LIMS) of Clinical Microbiology Laboratory at Makerere University College of Health Sciences, located in MNRH complex: 130 samples in 2014, 67 in 2015, 67 in 2016, 81 in 2017, and 85 in 2018.

\subsection{Setting}

\subsubsection{General Setting}

Uganda's public hospitals had a surgical volume of 36,670 cases in 2018, with women most commonly undergoing surgery $(78.3 \%)$, with a mean age of 26.9 years [21]. Overall case distribution was $69 \%$ obstetrics / gynaecology, $24 \%$ general surgery, $4 \%$ orthopaedics, and $3 \%$ other subspecialties [21].

\subsubsection{Specific Setting}

Laboratory analysis of samples was carried out at the Clinical Microbiology Laboratory at Makerere University College of Health Sciences, located in MNRH complex. This laboratory receives and processes clinical samples from MNRH wards. We utilized data from all bacteriologically positive samples obtained from obstetrics/gynaecology, general surgery, and orthopaedic wards of MNRH. To perform this, our study accessed the LIMS of the Clinical Microbiology Laboratory. This clinical laboratory is accredited by the College of American Pathologists. MNRH was founded in 1913 and is a teaching hospital for Makerere University College of Health Sciences. It also serves as a general hospital for the Kampala metropolitan area, whose population is 1.3 million [17]. Established hospital bed capacity is 1790, offering both public and private health services [22].

\subsection{Variables and Data Acquisition}

The following variables were extracted and included in the study: sample type, name of the surgical ward, and bacterial culture, as well as sensitivity of test results. Antibiotic classes and abbreviations can be seen in Table 1 .

Table 1. The antibiotic classes and their respective abbreviations.

\begin{tabular}{cc}
\hline Antibiotic Classes & Antibiotics (Abbreviation) \\
Penicillins & $\begin{array}{c}\text { penicillin (PEN), ampicillin (AMP), piperacillin } \\
\text { (PIPERAC), piperacillin-tazobactam (TPZ), oxacillin } \\
\text { (OXACILL), amoxicillin-clavulanic acid (AMOXCLAV) }\end{array}$ \\
\hline Third generation cephalosporins & cefotaxime (CEFOTAX), ceftriaxone (CEFTRIA), \\
ceftazidime (CAZ) \\
\hline Macrolides & erythromycin (ERYTH) \\
\hline Second generation cephalosporins & cefuroxime (CXM) \\
\hline Sulphonamides & trimethoprim-sulfamethoxazole (SXT)
\end{tabular}


Table 1. Cont.

\begin{tabular}{cc}
\hline Antibiotic Classes & Antibiotics (Abbreviation) \\
\hline Aminoglycoside & $\begin{array}{c}\text { gentamicin (GENTA), amikacin (AMIKACI), high-level } \\
\text { aminoglycoside (HLAR), }\end{array}$ \\
\hline Tetracycline & tetracycline (TETRAC) \\
\hline Phenicols & chloramphenicol (CHLORAM) \\
\hline Fluoroquinolones & ciprofloxacin (CIPROFL) \\
\hline Carbapenems & meropenem (MEROP), imipenem (IMIPENE) \\
\hline Lincosamides & clindamycin (CLINDA) \\
\hline Oxazolidinones & colistin (CT) \\
\hline Polymyxin E & nitrofurantoin (NITROFU) \\
\hline Nitrofurans & vancomycin (VANCO) \\
\hline Glycopeptide & cefepime (CEFEPIM) \\
\hline Fourth-generation cephalosporin &
\end{tabular}

\subsection{Data Collection}

The study data were collected by trained research assistants from the LIMS and entered into Microsoft Excel software for Windows 10. For data validity, all collected data were crosschecked for errors.

\subsection{Microbiological Process of Phenotypic Resistance}

Our study included laboratory data on the antimicrobial susceptibility profiles of Gram-negative and Gram-positive bacterial isolates from patient samples that had originated from the obstetrics/gynaecology, general surgery, and orthopaedics wards of Mulago National Referral Hospital between 2014 and 2018 (Table 2). All samples had been bacteriologically processed according to the laboratory's standard operating procedures. The organisms were identified using conventional biochemical tests according to the laboratory's standard operating procedures and/or the Phoenix Automated Machine (Becton-Dickinson, Franklin Lakes, NJ, USA), according to the manufacturer's instructions. Antimicrobial susceptibility testing was performed using the Kirby Bauer disc diffusion method, according to procedures outlined in the Clinical and Laboratory Standards Institute (CLSI), while using known control bacterial strains [23].

Table 2. Culture samples collected from patients attending the surgical wards of Mulago National Referral Hospital, Uganda, from 2014 to 2018.

\begin{tabular}{ccc}
\hline Sample & Frequency $(\mathbf{n}=\mathbf{4 2 8})$ & Percentage (\%) \\
\hline Tracheal aspirate & 156 & $(36.5)$ \\
Pus swab & 120 & $(28.0)$ \\
Blood & 88 & $(20.6)$ \\
Urine & 22 & $(5.1)$ \\
Pus aspirate & 20 & $(4.7)$ \\
Catheter tip & 13 & $(3.0)$ \\
High vaginal swab & 3 & $(0.7)$ \\
Ear swab & 3 & $(0.7)$ \\
Wound swab & 2 & $(0.5)$ \\
Stool & 1 & $(0.2)$ \\
\hline
\end{tabular}




\subsection{Data Analysis}

The data extracted from LIMS were cleaned and analyzed using Microsoft Excel 2016 (Microsoft Corporation, Albuquerque, NM, USA) and STATA 14.0 statistical software (StataCorp, College Station, TX, USA). Descriptive analyses including frequencies, percentages, and means were performed. Cross-tabulations were used to determine the percentages of samples that were susceptible towards each antibiotic. These percentages were presented in the form of tables and figures. Resistance percentage categories were assigned for this study to determine the trends of resistance towards the antibiotics. $\mathrm{Chi}^{2}$ tests were used to assess the significance of differences in resistance towards the selected antibiotics for 2014, 2016, and 2018. Differences with $p$-values less than 0.05 at a $95 \%$ confidence interval were considered significant.

\section{Results}

Table 2 shows the sources and proportions of culture samples that were tested. Tracheal aspirates, pus swabs, and blood were the most common sources.

\subsection{Bacterial Isolates from the Samples}

Bacterial isolates were both Gram-positive and Gram-negative bacteria (Enterobacteriaceae and non-Enterobacteriaceae). The majority of the Enterobacteriaceae were Klebsiella spp. $(21.7 \%)$, followed by E. coli $(15.9 \%)$, while the majority of the non-Enterobacteriaceae were Acinetobacter spp. (17.5\%). Gram-positives were mostly Staphylococcus spp. (12.4\%), followed by Enterococcus spp. (6.1\%) (Table 3).

Table 3. Bacteria isolated from samples taken from patients attending the surgical wards of Mulago National Referral Hospital, Uganda, from 2014 to 2018.

\begin{tabular}{cccc}
\hline Classification & Frequency $(\mathbf{n}=\mathbf{4 2 8})$ & Percentage (\%) \\
\hline & Klebsiella spp. & 93 & $(21.7)$ \\
& E. coli & 68 & $(15.9)$ \\
Gram-negative & Proteus spp. & 19 & $(4.4)$ \\
Enterobacteriaceae $(\mathrm{n}=216)$ & Enterobacter spp. & 18 & $(4.2)$ \\
& Citrobacter spp. & 11 & $(2.6)$ \\
& Serratia marcescens & 4 & $(0.9)$ \\
& Providencia spp. & 3 & $(0.7)$ \\
\hline Gram-negative & Acinetobacter spp. & 75 & $(17.5)$ \\
non-Enterobacteriaceae $(\mathrm{n}=122)$ & Pseudomonas spp. & 47 & $(11.0)$ \\
\hline & Staphylococcus spp. & 53 & $(12.4)$ \\
Gram-positive & Enterococcus spp. & 26 & $(6.1)$ \\
$(\mathrm{n}=90)$ & Streptococcus spp. & 9 & $(2.1)$ \\
& Corynebacteria & 2 & $(0.5)$ \\
\hline
\end{tabular}

\subsection{Antimicrobial Susceptibility Profiles of Bacterial Isolates during 2014, 2016, and 2018}

Figure 1 shows the sensitivities and resistance patterns of antibiotics from 2014, 2016, and 2018. There were minor fluctuations in antibiotic resistance year by year, but, in general, certain classes of antibiotics tended to demonstrate more resistance than others. 


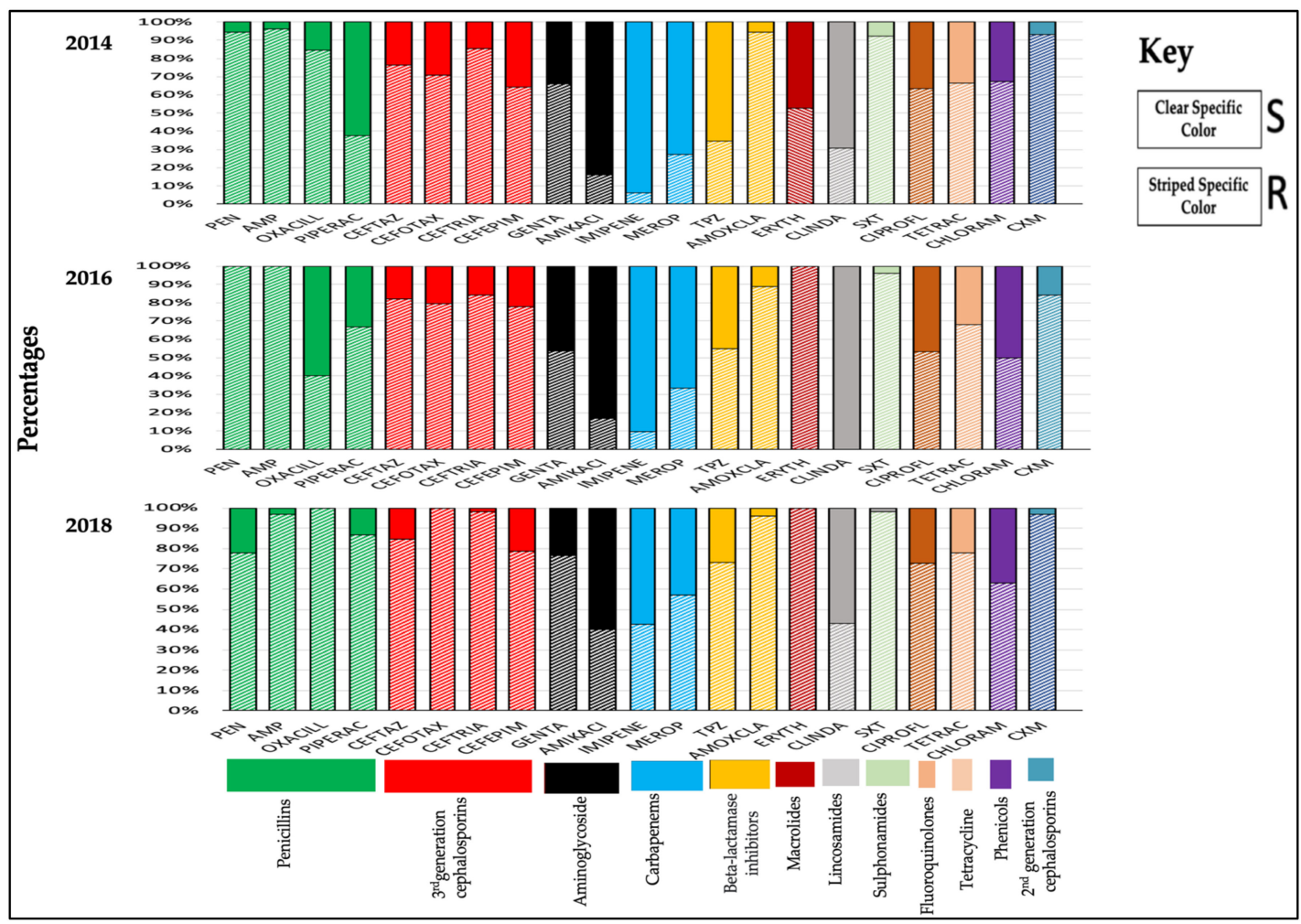

Figure 1. Antimicrobial susceptibility profiles of bacterial isolates from surgical wards of Mulago National Referral Hospital, Uganda, from 2014, 2016, and 2018.

\subsection{Antimicrobial Resistance Trends over the Years}

Among Gram-positive isolates, there were fluctuations in resistance, especially in 2016, but the trend was for an overall increase from 2014 to 2018 (Figure 2A) and (Table 4). Similarly, there was a clear trend for increased resistance among Gram-negative bacteria for virtually all antibiotics (Figure 2B) and (Table 5).
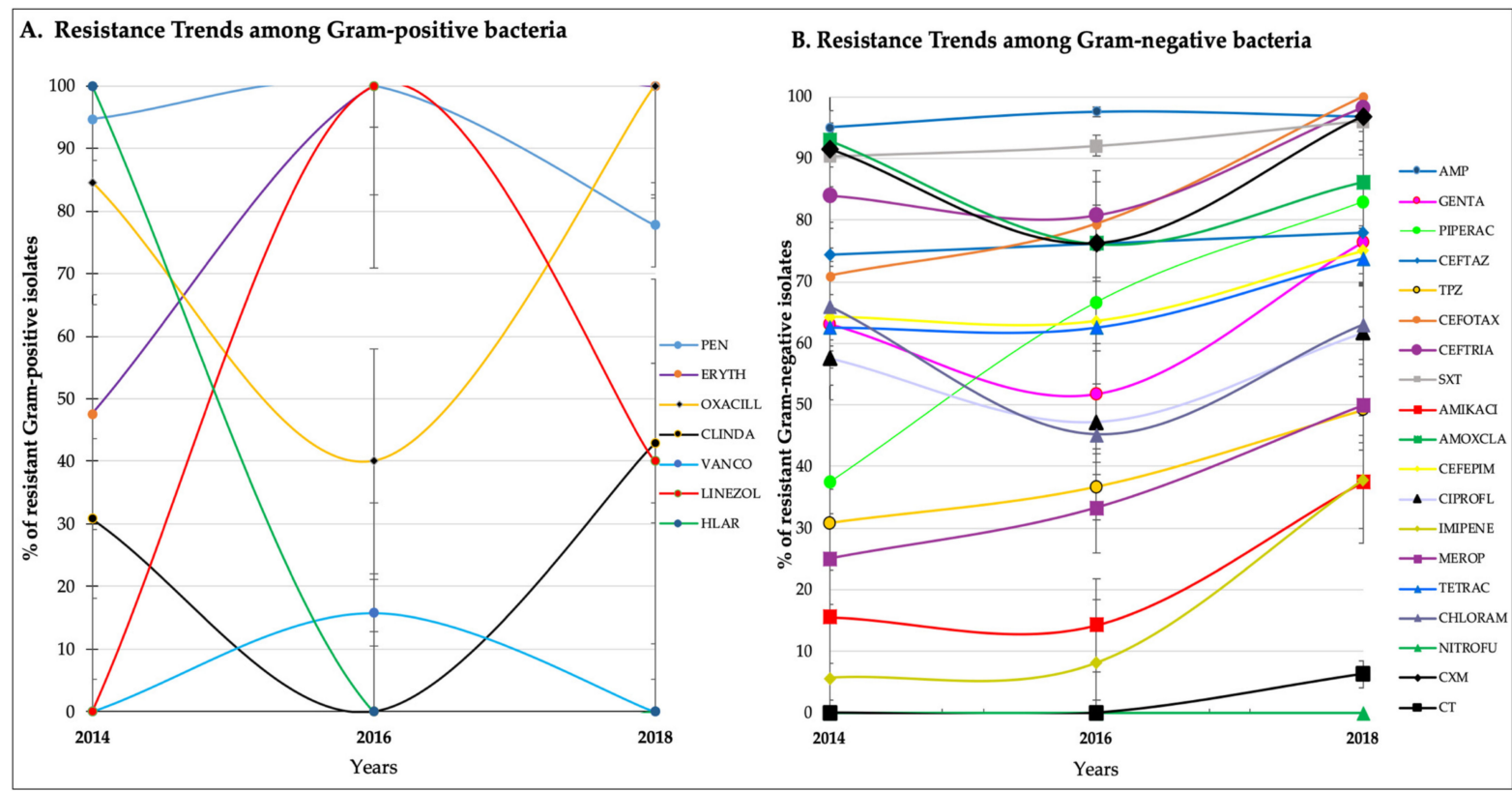

Figure 2. (A) Antimicrobial resistance trends among Gram-positive bacterial isolates for 2014, 2016, and 2018; (B) Antimicrobial resistance trends among Gram-negative bacterial isolates of samples collected from the surgical wards of MNRH, Uganda, for 2014, 2016, and 2018. 
Table 4. Resistance of Gram-positive isolates towards antibiotics from 2014 to 2018.

\begin{tabular}{|c|c|c|c|c|c|c|c|}
\hline Antibiotic/Years & $\begin{array}{c}2014 \\
\text { n (\%) }\end{array}$ & $\begin{array}{c}2015 \\
\text { n (\%) }\end{array}$ & $\begin{array}{c}2016 \\
\text { n (\%) }\end{array}$ & $\begin{array}{c}2017 \\
\text { n (\%) }\end{array}$ & $\begin{array}{c}2018 \\
\text { n (\%) }\end{array}$ & $\mathrm{Chi}^{2}$ Value for Trends & $p$-Value \\
\hline Penicillin & $18(94.7)$ & $16(88.9)$ & $10(100.0)$ & $7(77.8)$ & $7(77.8)$ & 2.13 & 0.144 \\
\hline Erythromycin & $10(47.6)$ & $13(68.4)$ & $21(100.0)$ & $8(72.7)$ & $9(100.0)$ & 9.94 & 0.002 \\
\hline Oxacillin & $11(84.6)$ & $6(40.0)$ & $3(60.0)$ & $2(40.0)$ & $4(100.0)$ & 0.03 & 0.871 \\
\hline Clindamycin & $4(30.8)$ & $2(18.2)$ & $0(0.0)$ & $1(14.3)$ & $3(42.9)$ & 0.01 & 0.938 \\
\hline Linezolid & $0(0.0)$ & $1(50.0)$ & $3(100)$ & $0(0.0)$ & $2(40.0)$ & 0.08 & 0.778 \\
\hline HLAR & $2(100.0)$ & $2(100.0)$ & - & $2(50.0)$ & $0(0.0)$ & 3.56 & 0.059 \\
\hline
\end{tabular}

There was a statistically significant increased trend in resistance towards erythromycin from $47.6 \%$ in 2014 to $100 \%$ in $2018\left(x^{2}\right.$ trends $=9.94$, $p$-value $<0.001)$. The bold shows statistically significant value.

Table 5. Resistance of Gram-negative isolates towards antibiotics from 2014 to 2018.

\begin{tabular}{|c|c|c|c|c|c|c|c|}
\hline Antibiotic/Year & 2014 n (\%) & 2015 n (\%) & 2016 n (\%) & 2017 n (\%) & 2018 n (\%) & $\begin{array}{l}\mathrm{Chi}^{2} \text { Value } \\
\text { for Trends }\end{array}$ & $p$-Value \\
\hline Ampicillin & $76(95.0)$ & $34(100.0)$ & $41(97.6)$ & $44(97.8)$ & $30(96.8)$ & 0.27 & 0.603 \\
\hline Gentamicin & $70(63.1)$ & $19(61.3)$ & $30(51.7)$ & $42(57.5)$ & $55(76.4)$ & 0.72 & 0.397 \\
\hline Piperacillin & $3(37.5)$ & $7(58.3)$ & $6(66.7)$ & $16(64.0)$ & $39(83.0)$ & 9.58 & 0.002 \\
\hline Cefotaxime & $58(74.4)$ & $9(42.9)$ & $32(76.2)$ & $30(75.0)$ & $39(78.0)$ & 1.72 & 0.189 \\
\hline Piperacillin-Tazobactam & $8(30.8)$ & $7(21.9)$ & $11(36.7)$ & $19(41.3)$ & $30(49.2)$ & 14.83 & $<0.001$ \\
\hline Cefotaxime & $22(71.0)$ & $3(33.3)$ & $27(79.4)$ & $27(93.1)$ & $7(100)$ & 8.02 & 0.005 \\
\hline Ceftriaxone & $58(84.1)$ & $11(61.1)$ & $21(80.8)$ & $34(89.5)$ & $53(98.2)$ & 9.95 & 0.008 \\
\hline Trimethoprim-sulfamethoxazole & $74(90.2)$ & $35(87.5)$ & $23(92.0)$ & $39(90.7)$ & $49(96.1)$ & 1.34 & 0.245 \\
\hline Amikacin & $6(15.6)$ & $6(15.6)$ & $4(14.3)$ & $9(20.9)$ & $18(37.5)$ & 7.01 & 0.008 \\
\hline Amoxicillin clavulanic acid & $52(92.9)$ & $31(93.9)$ & $16(76.2)$ & $24(80.0)$ & $25(86.2)$ & 0.84 & 0.361 \\
\hline Cefepime & $9(64.3)$ & $4(40.0)$ & $7(63.6)$ & $24(63.2)$ & $33(75.0)$ & 2.25 & 0.134 \\
\hline Ciprofloxacin & $38(57.6)$ & $18(42.9)$ & $17(47.2)$ & $38(58.5)$ & $37(61.7)$ & 2.01 & 0.156 \\
\hline Imipenem & $4(5.7)$ & $1(3.3)$ & $3(8.1)$ & $14(22.6)$ & $25(37.9)$ & 32.29 & $<0.001$ \\
\hline Meropenem & $3(25.0)$ & $2(100.0)$ & $1(33.3)$ & $1(14.3)$ & $4(50.0)$ & 0.36 & 0.547 \\
\hline Tetracycline & $10(62.5)$ & $8(47.1)$ & $15(62.5)$ & $8(44.4)$ & $14(73.7)$ & 0.56 & 0.453 \\
\hline Chloramphenicol & $31(66.0)$ & $22(50.0)$ & $19(45.2)$ & $28(59.6)$ & $17(63.0)$ & 0.03 & 0.863 \\
\hline Nitrofurantoin & $0(0.0)$ & $0(0.0)$ & - & $2(22.2)$ & $0(0.0)$ & 0.84 & 0.358 \\
\hline Cefuroxime & $54(91.5)$ & $15(53.6)$ & $16(76.2)$ & $33(89.2)$ & $30(96.8)$ & 1.87 & 0.171 \\
\hline Colistin & - & $0(0.0)$ & $0(0.0)$ & $0(0.0)$ & $1(6.25)$ & 0.49 & 0.483 \\
\hline
\end{tabular}

Note: * $p$-value $<0.05$. There was a statistically significant increased trend in resistance of the isolates towards piperacillin $\left(x^{2}\right.$ trends $=9.58$, $p$-value $=0.02) ; \operatorname{TPZ}\left(\mathrm{x}^{2}\right.$ trends $=14.83, p$-value $\left.<0.001\right)$; cefotaxime $\left(\mathrm{x}^{2}\right.$ trends $=8.02, p$-value $\left.=0.005\right)$; ceftriaxone $\left(\mathrm{x}^{2}\right.$ trends $=9.95$, $p$-value $=0.008)$; amikacin $\left(\mathrm{x}^{2}\right.$ trends $=7.01, p$-value $\left.=0.008\right)$; and imipenem $\left(\mathrm{x}^{2}\right.$ trends $=36.4, p$-value $\left.<0.001\right)$ from the year 2014 to 2018. The bold shows statistically significant value.

\section{Discussion}

This was the first study in Uganda to document antimicrobial resistance trends over five years in a tertiary hospital's surgical ward. It showed a high level of resistance to many commonly prescribed antibiotics and a trend of increasing resistance to all antibiotics over five years.

This is very important because, in the absence of easily accessible laboratory-based bacteriological culture and sensitivity testing, clinicians will continue to prescribe antibiotics empirically. Knowing which classes of antibiotics have high resistance patterns becomes crucial for choosing antibiotics that are effective in treating infections. This study reinforces the practice that regular surveillance of antibiotic resistance is required to provide timely resistance pattern information to clinicians. It also underscores efforts to reduce AMR by adhering to good infection prevention and control practices.

Our findings are in agreement with a cross-sectional study from 2011 in MNRH examining bacterial isolates from surgical wards, which reported a $100 \%$ resistance to ampicillin, piperacillin, and tetracycline, and a 3.9\% resistance to imipenem [24]. In other African studies, hospital-wide HAI prevalence varied between $2.5 \%$ and $14.8 \%$ in Algeria, Burkina Faso, Senegal, and the United Republic of Tanzania [3]. A study in Ethiopia from 
March to July 2015 observed that the prevalence of HAI was high in teaching hospitals, with surgical site infections and pneumonia being the most common [25]. This study is relevant since MNRH is the teaching hospital of Makerere University.

Previous studies on AMR analysis at MNRH [26-29] did not perform AMR trend analysis in the surgical wards. However, in Rwanda, which borders Uganda to the south west, a five-year antimicrobial susceptibility trend study among bacterial isolates from a tertiary health-care facility in Kigali, from 1 January 2009 to 31 December 2013, found high rates of resistance by Gram-negative bacteria to cephalosporins, and rising rates of resistance to last-option drugs such as imipenem and colistin [30]. Furthermore, in this study, colistin resistance was $18.5 \%$ and $3 \%$ against Acinetobacter spp. and Pseudomonas spp., respectively, whereas imipenem resistance was $54.8 \%$ and $15.7 \%$, respectively, for the same organisms [30]. It may be important to note that, while Gram-negative bacteria are more sensitive to carbapenems compared to other beta-lactams [31], over the years, carbapenem resistance has increased. Therefore, even if this remains the choice of antibiotic for drug resistant bacteria, increasing resistance to this antibiotic poses a threat to the management of multiple drug resistant (MDR) organisms.

Our study has several operational implications. First, the rise and spread of antibioticresistant bacteria in hospital settings continue to reduce antibiotic options, particularly for infections involving antibiotic-resistant Gram-positive or Gram-negative bacteria. This has resulted in the increased use of last-option antibiotics, often reserved for life-threatening infections, a practice that may accelerate the development of resistance. Second, the growing trend of resistance should be a trigger to strengthen antibiotic stewardship and maximize infection prevention and control (IPC) practices to safeguard the remaining effective antibiotics. The need for local antibiotics stewardship programs cannot be overemphasized. Third, it points towards increasing laboratory capacity to perform bacteriological culture and sensitivity in a timely manner, so that antibiotics can be chosen based on evidence and not just clinical intuition. Finally, capacity needs to be built for routine AMR trend analysis in clinical microbiology laboratories to support the Ministry of Health in creating antibiotic stewardship programs.

The strengths of this study include the following: (i) data were available over a five-year period from the surgical wards of MNRH to provide a trend analysis; (ii) the bacteriological culture results came from the Clinical Microbiology Laboratory at Makerere University College of Health Sciences, which is a College of American Pathologists (CAP) accredited established facility with good quality control practices; and (iii) the study followed Strengthening the Reporting of Observational Studies in Epidemiology (STROBE) guidelines [32].

There were also some limitations, which included the following: (i) a lack of timely culture and sensitivity information available to clinicians; (ii) a small number of surgical site specimens at the time of the study; (iii) the number of sample types included in this study was constrained by the number of samples submitted to the study laboratory during the period under consideration; therefore, there was an inherent selection bias; and (iv) there was a high proportion of tracheal aspirate samples, which are not typically surgical site infections. Lastly, our analysis did not include an intermediate susceptibility category; we specifically looked at "Resistance" or "Susceptible" categories.

\section{Conclusions}

This study showed that, disturbingly, AMR increased across all antibiotic classes over five years in Mulago National Reference Hospital. It strongly suggests that ongoing surveillance be established to provide clinicians with up-to-date antibiograms to ensure that appropriate antimicrobial choices are based on the latest evidence. It also suggests that the laboratory introduces timely culture and sensitivity testing to properly target HAI pathogens. 
Author Contributions: Conceptualization, G.M., D.A.; methodology, G.M., D.A., H.D., P.O., E.M.K., W.E., and A.R.; validation, G.M., D.A., and D.B.; formal analysis, G.M., D.A., D.B., and F.E.K.; investigation, G.M., D.A., J.K., and M.L.; resources, G.M.; data curation, G.M., D.A., and D.B.; writing-original draft preparation, G.M. and D.A.; writing-review and editing, G.M., D.A., D.B., I.S., F.E.K., H.D., P.O., E.M.K., W.E., A.R., J.K., M.L., and H.K.; visualization, G.M., D.A., and D.B.; supervision, H.K. and F.E.K.; project administration, G.M.; funding acquisition, G.M. All authors have read and agreed to the published version of the manuscript.

Funding: This SORT IT AMR programme was funded by National Institute of Health Research, Department of Health and Social Care of the United Kingdom, and supported by implementing partners.

Institutional Review Board Statement: The study was conducted according to the guidelines of the Declaration of Helsinki and approved by the Institutional Review Board or Ethics Committee of the School of Biomedical Sciences College of Health Sciences Makerere University (SBS-REC); SBS-769, approved on 24 June 2020. This work was also approved by the Ethics Advisory Group of the International Union Against Tuberculosis and Lung Disease (The Union), with EAG number: 70/19 approval number granted on 29 October 2019.

Informed Consent Statement: As this was a record review study with no patient identifiers, the issue of informed patient consent did not apply.

Data Availability Statement: Not applicable.

Acknowledgments: This research was conducted through the Structured Operational Research and Training Initiative (SORT IT), a global partnership coordinated by TDR, the Special Programme for Research and Training in Tropical Diseases at the World Health Organization (TDR). The specific SORT IT program that led to these publications included a partnership of TDR with WHO Country offices of Ghana, Sierra Leone, and Uganda, and was implemented along with The Tuberculosis Research and Prevention Center Non-Governmental Organization, Armenia; The International Union Against Tuberculosis and Lung Diseases, Paris and South East Asia offices; Institute of Tropical Medicine, Antwerp, Belgium; Sustainable Health Systems, Freetown, Sierra Leone; Médecins Sans Frontières-Luxembourg (LuxOR) Centre National de Formation et de Recherche en Santé Rurale de Maferinyah, Guinea; Bahir Dar University Bahir Dar, Ethiopia, Makerere and Lira Universities, Uganda and the University of Salford, United Kingdom. Lastly, we thank all laboratory staffs of the Clinical Microbiology Laboratory of the department of Medical Microbiology at Makerere University College of Health Sciences for their role in routinely receiving and processing these laboratory specimens.

Conflicts of Interest: The authors declare that there are no conflict of interest regarding the publication of this work. The funders had no role in the design of the study; in the collection, analyses, or interpretation of data; in the writing of this manuscript; or in the decision to publish the results.

Open Access Statement and Disclaimer: In accordance with WHO's open-access publication policy for all work funded by WHO or authored/co-authored by WHO staff members, WHO retains the copyright of this publication through a Creative Commons Attribution IGO license (http:// creativecommons.org/licenses/by/4.0/igo/legalcode; accessed on 14 April 2021) which permits unrestricted use, distribution and reproduction in any medium provided the original work is properly cited. There should be no suggestion that WHO endorses any specific organization, products or services. The views expressed in this article are those of the authors and do not necessarily reflect those of their affiliated institutions. The use of the WHO logo is not permitted. This notice should be preserved along with the article's original URL.

\section{References}

1. Irek, E.O.; Amupitan, A.A.; Obadare, T.O.; Aboderin, A.O. A systematic review of healthcare-associated infections in Africa: An antimicrobial resistance perspective. Afr. J. Lab. Med. 2018, 7, 9. [CrossRef] [PubMed]

2. Sengupta, S.; Barman, P.; Lo, J. Opportunities to overcome implementation challenges of infection prevention and control in low-middle income countries. Curr. Treat. Options Infect. Dis. 2019, 11, 267-280. [CrossRef]

3. WHO. Health-Care-Associated Infection in Africa: A Systematic Review; WHO World Health Organization: Geneva, Switzerland, 2011. Available online: https://www.who.int/bulletin/volumes/89/10/11-088179/en/ (accessed on 6 March 2021).

4. Greco, D.; Magombe, I. Hospital acquired infections in a large north Ugandan hospital. J. Prev. Med. Hyg. 2011, 52, 55-58. [PubMed] 
5. Hope, D.; Ampaire, L.; Oyet, C.; Muwanguzi, E.; Twizerimana, H.; Apecu, R.O. Antimicrobial resistance in pathogenic aerobic bacteria causing surgical site infections in Mbarara regional referral hospital, Southwestern Uganda. Sci. Rep. 2019, 9, 1-10. [CrossRef]

6. Antimicrobial Resistance. Available online: https://www.who.int/news-room/fact-sheets/detail/antimicrobial-resistance (accessed on 28 February 2021).

7. Ampaire, L.; Muhindo, A.; Orikiriza, P.; Mwanga-Amumpaire, J.; Bebell, L.; Boum, Y. A review of antimicrobial resistance in East Africa. Afr. J. Lab. Med. 2016, 5, 432. [CrossRef] [PubMed]

8. SSI-Surveillance-Protocol.pdf. Available online: https://www.who.int/infection-prevention/tools/surgical/SSI-surveillanceprotocol.pdf?ua=1 (accessed on 28 February 2021).

9. Dessie, W.; Mulugeta, G.; Fentaw, S.; Mihret, A.; Hassen, M.; Abebe, E. Pattern of bacterial pathogens and their susceptibility isolated from surgical site infections at selected referral hospitals, Addis Ababa, Ethiopia. Int. J. Microbiol. 2016, 2016, 1-8. [CrossRef] [PubMed]

10. Seni, J.; Najjuka, C.F.; Kateete, D.P.; Makobore, P.; Joloba, M.L.; Kajumbula, H.; Kapesa, A.; Bwanga, F. Antimicrobial resistance in hospitalized surgical patients: A silently emerging public health concern in Uganda. BMC Res. Notes 2013, 6, 298. [CrossRef] [PubMed]

11. Manyahi, J.; Matee, M.I.; Majigo, M.; Moyo, S.; Mshana, S.E.; Lyamuya, E.F. Predominance of multi-drug resistant bacterial pathogens causing surgical site infections in Muhimbili national hospital, Tanzania. BMC Res. Notes 2014, 7, 500. [CrossRef]

12. Leaper, D.J.; Van Goor, H.; Reilly, J.; Petrosillo, N.; Geiss, H.K.; Torres, A.J.; Berger, A. Surgical site infection-A European perspective of incidence and economic burden. Int. Wound J. 2004, 1, 247-273. [CrossRef] [PubMed]

13. GoU_AMR-NAP.pdf. Available online: https://cddep.org/wp-content/uploads/2018/12/GoU_AMR-NAP.pdf (accessed on 3 April 2021).

14. World Health Organization. Antimicrobial Resistance: Global Report on Surveillance; World Health Organization: Geneva, Switzerland, 2014.

15. GtR. Available online: https://gtr.ukri.org/projects?ref=MR\%2FS004793\%2F1 (accessed on 3 April 2021).

16. The University of Edinburgh. Uganda Fleming Fellowships in AMR Surveillance at the University of Edinburgh. Available online: https://www.ed.ac.uk/edinburgh-infectious-diseases/news/news/uganda-fleming-fellowships-in-amr-surveillance (accessed on 3 April 2021).

17. Uganda Population (2021)-Worldometer. Available online: https://www.worldometers.info/world-population/ugandapopulation/ (accessed on 1 March 2021).

18. Micah, A.E.; Su, Y.; Bachmeier, S.D.; Chapin, A.; Cogswell, I.E.; Crosby, S.W.; Cunningham, B.; Harle, A.C.; Maddison, E.R.; Moitra, M.; et al. Health sector spending and spending on HIV/AIDS, tuberculosis, and malaria, and development assistance for health: Progress towards Sustainable Development Goal 3. Lancet 2020, 396, 693-724. [CrossRef]

19. Uganda GDP per Capita | 1982-2019 Data | 2020-2021 Forecast | Historical | Chart. Available online: https:/ / tradingeconomics. com/uganda/gdp-per-capita (accessed on 1 March 2021).

20. Institute for Health Metrics and Evaluation. Uganda. 2015. Available online: http:/ /www.healthdata.org/uganda (accessed on 1 March 2021).

21. Albutt, K.; Punchak, M.; Kayima, P.; Namanya, D.B.; Shrime, M.G. Operative volume and surgical case distribution in Uganda's public sector: A stratified randomized evaluation of nationwide surgical capacity. BMC Health Serv. Res. 2019, 19, 1-9. [CrossRef] [PubMed]

22. Mulago National Referral Hospital I EA Health. Available online: https://www.eahealth.org/directory/search/organisations / mulago-national-referral-hospital-0 (accessed on 27 February 2021).

23. Performance standards for antimicrobial susceptibility testing. 30th edition. CLSI Suppl. M100 2020, 40, 1-332.

24. Antibiotic Resistance in Uganda: Situation Analysis and Recommendations. Available online: https://www.cddep.org/wpcontent/uploads/2017/06/uganda_antibiotic_resistance_situation_reportgarp_uganda_0-1.pdf (accessed on 1 March 2021).

25. Yallew, W.W.; Takele, A.K.; Yehuala, F.M. Point prevalence of hospital-acquired infections in two teaching hospitals of Amhara region in Ethiopia. Drug Health Patient Saf. 2016, 8, 71-76. [CrossRef] [PubMed]

26. Kajumbula, H.; Fujita, A.W.; Meya, D.B.; Boulware, D.R.; Manabe, Y.C.; Mbabazi, O.; Najjuka, C.; Izale, C.; Akampurira, A.; Aisu, S.; et al. Antimicrobial drug resistance in blood culture isolates at a tertiary hospital, Uganda. Emerg. Infect. Dis. 2018, 24, 174-175. [CrossRef] [PubMed]

27. Wekesa, Y.N.; Namusoke, F.; Sekikubo, M.; Mango, D.W.; Bwanga, F. Ceftriaxone- and ceftazidime-resistant Klebsiella species, Escherichia coli, and methicillin-resistant Staphylococcus aureus dominate caesarean surgical site infections at Mulago Hospital, Kampala, Uganda. SAGE Open Med. 2020, 8. [CrossRef]

28. Aruhomukama, D.; Najjuka, C.F.; Kajumbula, H.; Okee, M.; Mboowa, G.; Sserwadda, I.; Mayanja, R.; Joloba, M.L.; Kateete, D.P. blaVIM- and blaOXA-mediated carbapenem resistance among Acinetobacter baumannii and Pseudomonas aeruginosa isolates from the Mulago hospital intensive care unit in Kampala, Uganda. BMC Infect. Dis. 2019, 19, 853. [CrossRef] [PubMed]

29. Kateete, D.P.; Nakanjako, R.; Okee, M.; Joloba, M.L.; Najjuka, C.F. Genotypic diversity among multidrug resistant Pseudomonas aeruginosa and Acinetobacter species at Mulago Hospital in Kampala, Uganda. BMC Res. Notes 2017, 10, 1-10. [CrossRef] [PubMed] 
30. Carroll, M.; Singer, D.; Ogbuagu, O.; Rangaiahagari, A.; Musabeyezu, E. Five-year antimicrobial susceptibility trends among bacterial isolates from a tertiary health-care facility in Kigali, Rwanda. Am. J. Trop. Med. Hyg. 2016, 95, 1277-1283. [CrossRef] [PubMed]

31. Papp-Wallace, K.M.; Endimiani, A.; Taracila, M.A.; Bonomo, R.A. Carbapenems: Past, present, and future. Antimicrob. Agents Chemother. 2011, 55, 4943-4960. [CrossRef] [PubMed]

32. Field, N.; Cohen, T.; Charlett, A.; Egger, M.; Green, J.; Vineis, P.; Abubakar, I.; Struelens, M.J.; Palm, D.; Cookson, B.; et al. Strengthening the reporting of molecular epidemiology for infectious diseases (Strome-ID): An extension of the strobe statement. Lancet Infect. Dis. 2014, 14, 341-352. [CrossRef] 